\title{
Automated modal tracking and fatigue assessment of a wind turbine based on continuous dynamic monitoring
}

\author{
Gustavo Oliveira ${ }^{1, a}$, Filipe Magalhães ${ }^{1}$, Álvaro Cunha ${ }^{1}$ and Elsa Caetano ${ }^{1}$ \\ ${ }^{1}$ Construct-ViBest, Faculty of Engineering, University of Porto - Portugal
}

\begin{abstract}
The paper describes the implementation of a dynamic monitoring system at a $2.0 \mathrm{MW}$ onshore wind turbine. The system is composed by two components aiming at the structural integrity and fatigue assessment. The first component enables the continuous tracking of modal characteristics of the wind turbine (natural frequency values, modal damping ratios and mode shapes) in order to detect abnormal deviations of these properties, which may be caused by the occurrence of structural damage. On the other hand, the second component allows the estimation of the remaining fatigue lifetime of the structure based on the analysis of the measured cycles of structural vibration.
\end{abstract}

\section{Introduction}

Wind power is one of the most attractive alternatives to conventional energy solutions. With the development of the technology, higher and slender multi-MW wind turbine systems have been installed worldwide, both at onshore and offshore. Current wind turbines are large structures, prone to dynamic problems and fatigue wear. In that sense, monitoring systems developed to remotely assess the condition of these structures could be very useful to attest the real condition of the wind turbine.

This papers presents a continuous dynamic monitoring system, under development by the Laboratory of Vibrations and Structural Monitoring (ViBest, www.fe.up.pt), with the purpose of identifying structural changes (i.e. damage) and estimating the fatigue damage condition of the wind turbine. The monitoring system is based on Operational Modal Analysis (OMA) techniques [1], in which the modal properties of the structure (natural frequencies, modal damping ratios and mode shapes) are continuously tracked over the different operating conditions of the wind turbine. This strategy has already proven to be suitable to be installed both in onshore and offshore [2].

The first part of the paper introduces some results obtained with the continuous identification of the modal properties of the wind turbine. Some noticeable relationships between the modal properties and the operation/ environmental conditions are also shown.

The second part of the paper illustrates the application of the fatigue monitoring system to real data collected. In addition, the virtual sensors methodology, aiming at estimating the acceleration at unmeasured locations of the structure, is introduced in order to optimize the layout of the dynamic monitoring system.

\section{Description of the wind turbine and monitoring system}

The monitoring system is currently being tested at a 2.0 MW wind turbine with a variable-speed generator located at the north of Portugal. The turbine has a hub height of $80 \mathrm{~m}$ and an up-wind rotor with a diameter of $80 \mathrm{~m}$. The support structure is composed by a concrete slab foundation and a steel tower.

The implemented monitoring system consists of 9 uni-axial force balanced accelerometers, linked to a 24 bit digitizer and acquisition system. The sensors are installed along the tower according to Figure 1.

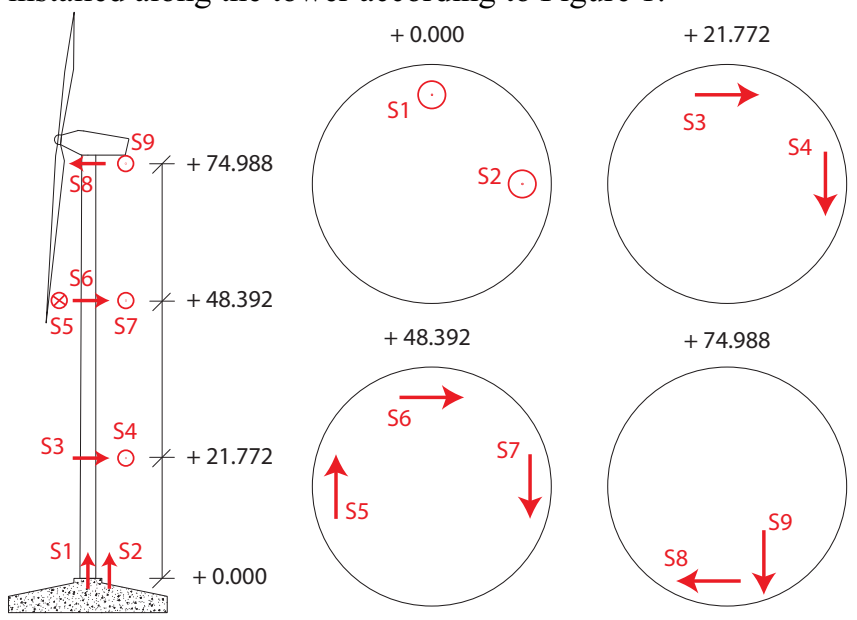

Figure 1. Position of the accelerometers at the different levels of the wind turbine.

\footnotetext{
${ }^{\mathrm{a}}$ Corresponding author: gustavo.oliveira@fe.up.pt
} 
The monitoring system records acceleration time series of $10 \mathrm{~min}$ with a sampling rate of $50 \mathrm{~Hz}$, subsequently decimated to $12.5 \mathrm{~Hz}$. The monitoring system is complemented by the operational and environmental data acquired by the wind turbine SCADA system.

The initial analysis of the collected acceleration data permits to identify a different vibration scenario of the turbine support structure according to the operating condition of the turbine. Figures 2 and 3 show the evolution of the root mean squared (RMS) values of the acceleration time series in the fore-aft (FA) direction at the 3 different measured levels during parked/ idling conditions (the rotor is stopped or slowly rotating) and operating conditions. It can be seen that, when the turbine is operating, the acceleration level at the $+48.392 \mathrm{~m}$ is clearly higher than at the other two locations. Indeed, the vibration levels at the top $(+74.988 \mathrm{~m})$ and $+21.772 \mathrm{~m}$ are similar. On the other hand, when the turbine is not operating, the level of vibration usually increases from the lower part to the top of the tower structure. Nevertheless, the vibration at the $+48.392 \mathrm{~m}$ sometimes exceeds the values recorded by the top sensor.

Firstly, it should be stated that the accelerations measured at the tower height of $48.392 \mathrm{~m}$ are highly influenced by the tower second bending mode (2 FA), whereas the ones measured at the tower top are essentially influenced by the first mode, with a considerably lower natural frequency. Consequently, if dynamic displacements were measured, the highest values would most likely occur at the tower top, as will be shown later. Anyhow, the highest accelerations at the intermediate level are certainly due to the excitation introduced by the blade passage in front of the tower (shadow effect), since the sensor at level $+48.392 \mathrm{~m}$ is located in a position where the second bending mode of the tower presents its maximum ordinate.

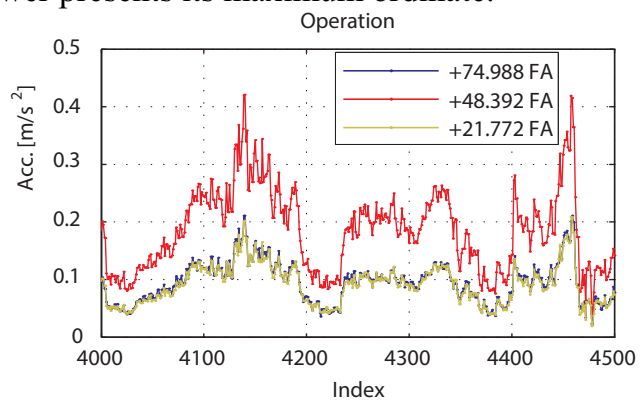

Figure 2. Evolution of the RMS values of acceleration during operating conditions

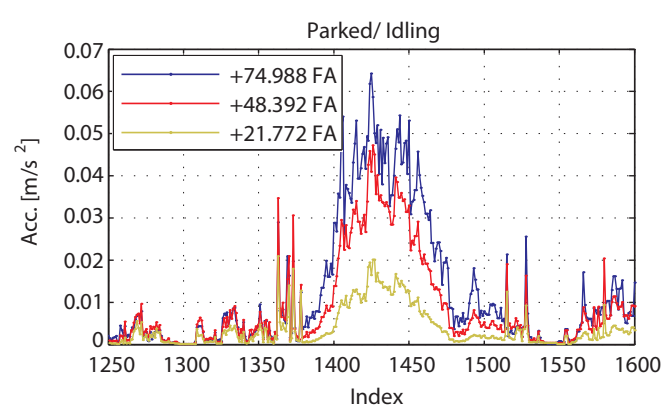

Figure 3. Evolution of the RMS values of acceleration during non-operating conditions

\section{Automated modal identification}

\subsection{Strategy followed}

The dynamic monitoring system is designed to identify the modal properties of the most important vibration modes of the wind turbine in an automated way [3].

The system is composed by an initial pre-processing, where the signals are filtered and re-sampled. The acceleration signals are then processed by output-only system identification algorithms in order to estimate the modal properties of the wind turbine. Two algorithms in the time-domain (SSI-COV [4] and SSI-DATA [5]) and one in the frequency-domain (p-LSCF [6] are used. The results obtained with these algorithms are usually presented in the form of stabilization diagrams. Figure 4 shows an example of a stabilization diagram obtained with p-LSCF algorithm.

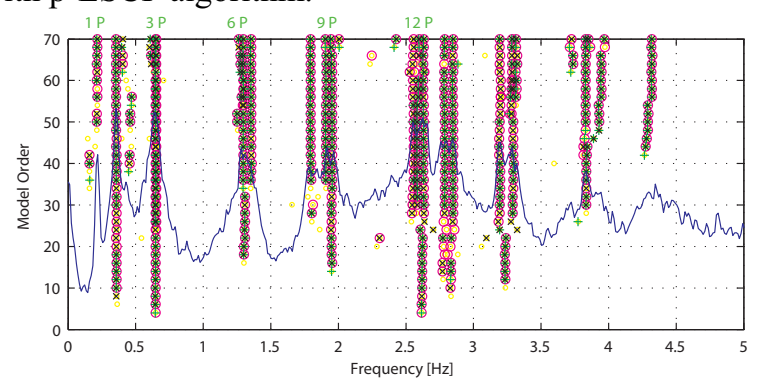

Figure 4. Stabilization diagram from a power production situation (the alignments of poles related to the harmonics are identified)

A methodology based on hierarquical clustering is then used for automated identification of the modal parameters [7]. This method is defined to aggregate poles with similar modal properties into the same cluster.

The modal properties of the clusters are then compared to reference properties in order to assign them to the corresponding vibration modes. Considering the different structural configurations and conditions of operation, 6 operating regimes were considered to define the modal reference properties of each tracked vibration mode. These regimes are presented in Table 1.

A representative configuration of the vibration modes to be tracked is presented in Figure 5. 
Table 1. Operating regimes considered for definition of the reference modal properties of the vibration modes.

\begin{tabular}{|c|c|}
\hline $\begin{array}{c}\text { Operating } \\
\text { regime }\end{array}$ & Wind turbine condition \\
\hline 1 & Parked or idling (with high pitch angle) \\
\hline 2 & $\begin{array}{c}\text { Parked or idling (with lower pitch angle, in } \\
\text { conditions to start operating) }\end{array}$ \\
\hline 3 & $\begin{array}{c}\text { Transition situation from non-operation to } \\
\text { operation (mean value of rotor speed } \\
\text { between } 0 \text { and the lowest operating rotor } \\
\text { speed) }\end{array}$ \\
\hline 4 & $\begin{array}{c}\text { Operating situation, defined by the lowest } \\
\text { operating rotor speed and the point where } \\
\text { the pitch angle starts to increase to avoid } \\
\text { excessive rotor torque values }\end{array}$ \\
\hline 5 & $\begin{array}{c}\text { Operating situation, between regime } 4 \text { and } \\
\text { the highest operating rotor speed }\end{array}$ \\
\hline 6 & Wind speed higher than cut-out speed \\
\hline
\end{tabular}
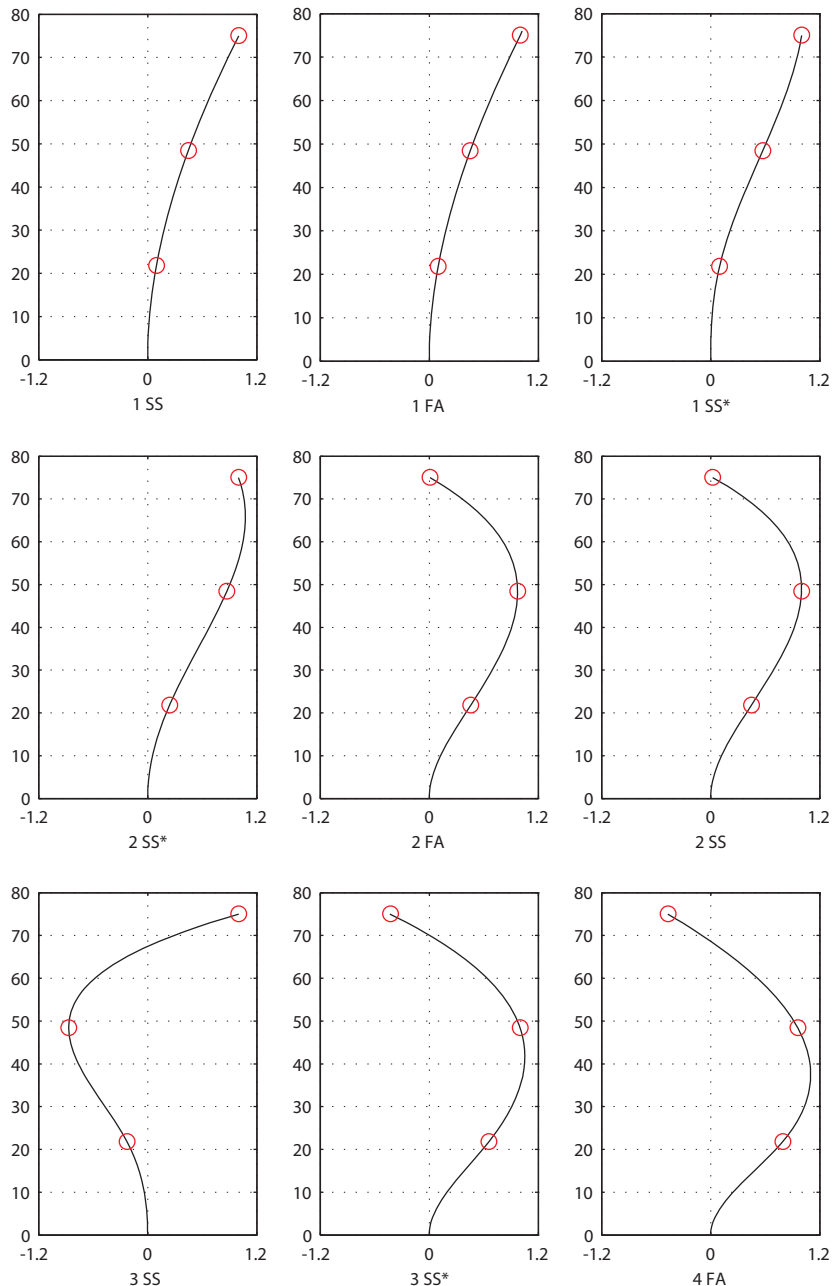

Figure 5. Mode shapes of the tracked vibration modes

\subsection{Modal identification results}

Figure 6 presents the tracked vibration modes in a Campbell diagram (frequency vs rotor rotation velocity), considering datasets collected during one year. In this figure, the vertical dashed lines separate the nonproduction situation (below $1 \mathrm{rpm}$ ), a transition state (rotor speed between 1 and $8.7 \mathrm{rpm}$ ) and the production regime (rotor speed higher than $8.7 \mathrm{rpm}$ ). It is seen that the 9 vibration modes were successfully identified across all the operating regimes of the wind turbine. Furthermore, it was possible to accurately separate the clusters referred to the vibration modes from the ones regarding the harmonics (in Figure 6, the diagonal dashed lines indicate the frequency of the multiples of the $3 \Omega$ harmonics, where $\Omega$ represents the rotor speed).

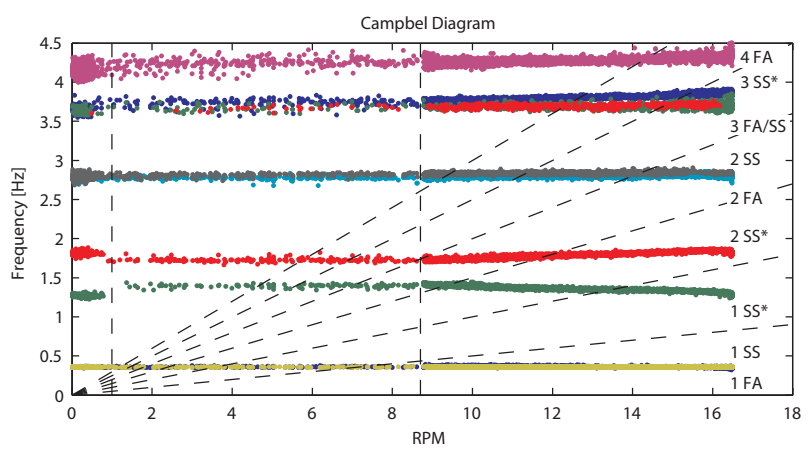

Figure 6. Campbell diagram with the tracked vibration modes

In Figure 6, some vibration modes show a clear dependency on the rotor rotation speed. These modes are identified with an asterisk (1 SS*, $2 \mathrm{SS}^{*}$ and $3 \mathrm{SS} *$ ). This behaviour is a characteristic of rotor vibration modes that, when in operation, develop two whirl modes: one forward and one backward mode. From a non-rotating reference (like the one from the installed sensors at the tower), the frequency values of these modes tend to increase and decrease, respectively for the forward and backward mode, with the increase of the rotor speed. This phenomenon is clearly shown in the zoom of Figure 7.

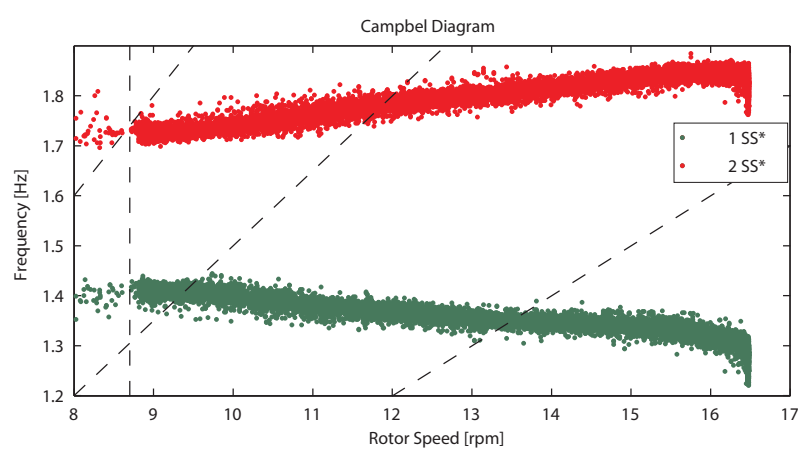

Figure 7. Zoom of the of the Campbell diagram for the modes 1 $\mathrm{SS}^{*}$ and $2 \mathrm{SS}^{*}$

Once the vibration modes are identified, it is possible to analyse the evolution of their properties throughout the different operating conditions. An important characteristic in the design of wind turbines is the damping of the $1^{\text {st }}$ tower bending mode in the FA direction (1 FA), since it has an important influence on the fatigue assessment of the structure. Figure 8 presents 
the evolution of modal damping ratios of the $1^{\text {st }}$ FA tower bending mode with the wind speed, showing all the estimated values and boxplots with the evolution of the median values and the $25 \%$ and $75 \%$ quartiles. It is clear that the damping values of the FA mode presents an important increase around a wind speed of $3 \mathrm{~m} / \mathrm{s}$. This situation corresponds to the start of operation of the turbine. Since this vibration mode presents large modal amplitude at the top of the tower and in the direction of the wind when in operation, the aerodynamic damping has a large influence in this mode. The damping values tend to increase with the wind speed until the blades pitch angle starts to increase (around $11 \mathrm{~m} / \mathrm{s}$ ). From this point, the increase rate decreases as consequence of the change on the aerodynamic configuration of the blades. The maximum damping values are obtained for wind speeds around $13 \mathrm{~m} / \mathrm{s}$, which corresponds to the rated wind speed of the turbine.

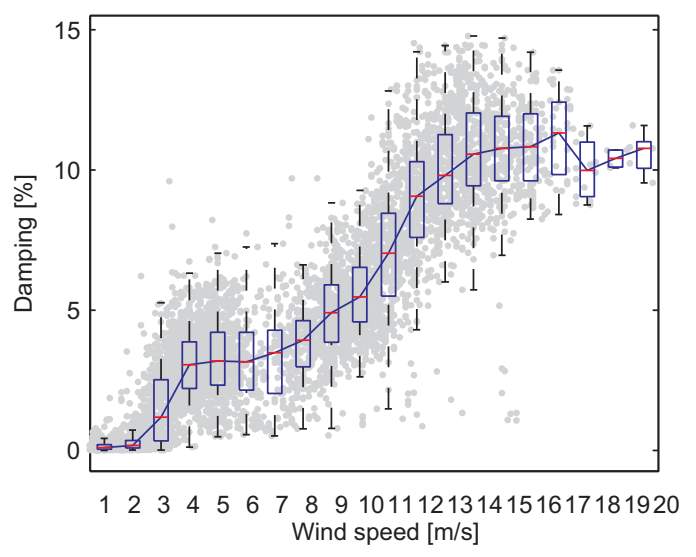

Figure 8. Damping ratio of the $1^{\text {st }}$ FA vibration mode vs wind speed

On the other hand, the evolution of the damping ratio of the $1^{\text {st }}$ tower bending mode in the side-side (SS) direction (1 SS) is shown is Figure 9. As observed, the damping values are consistently lower in this direction and much less dependent on the wind speed. This is due to the lower influence of the aerodynamic damping in this direction.

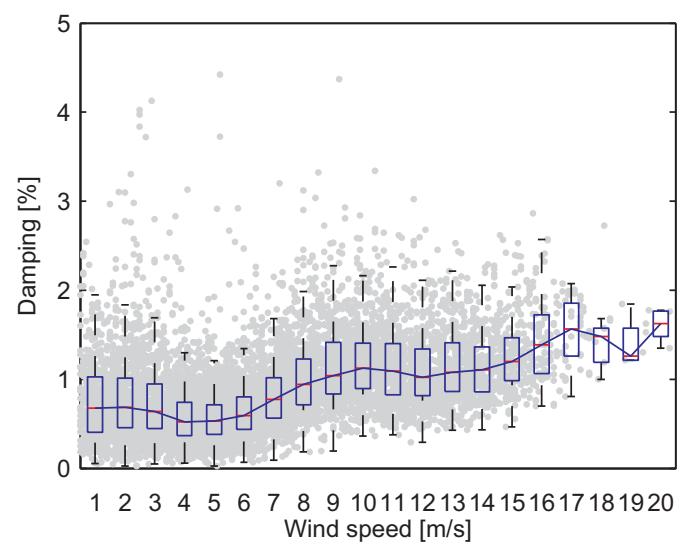

Figure 9. Damping ratio of the $1^{\text {st }}$ SS vibration mode vs wind speed

\section{Fatigue monitoring system}

Wind turbines are complex structural systems predominantly subjected to dynamic loads. It is thus important to estimate the remaining period of life of the turbine support structure in order to decide about repowering solutions or extension of the operating period.

The procedure implemented in the dynamic monitoring system for fatigue assessment uses the acceleration time series to estimate the stress condition at any point of the wind turbine support structure. The methodology adopted is based on the decomposition of the recorded accelerations in modal acceleration responses. Then, the fatigue stress due to each vibration mode or stationary response (due to harmonics) is computed and the real dynamic component of stress is obtained as a sum of all individual contributions.

\subsection{Procedure adopted for fatigue assessment}

The procedure for fatigue assessment of the wind turbine support structure based on acceleration time series was introduced and illustrated using a numerical model in [8]. It is based on the definition of a modal basis forward innovation model:

$$
\begin{gathered}
z_{m, k+1}=\Lambda_{d} z_{m, k}+K_{m} e_{k} \\
\ddot{y}_{k}=C z_{m, k}+e_{k}
\end{gathered}
$$

where $\Lambda_{d}$ and $C$ represent, respectively, the state-space matrix and output-matrix (in modal basis), $K_{m}$ indicates the Kalman filter gain (in modal basis), $z_{m}$ is the modal state sequence, $e_{k}$ is the white noise innovation sequence and $\ddot{y}_{k}$ is referred to the acceleration recorded at $k$ time step.

Once the model of equation (1) and (2) is defined, an estimate of the original acceleration time series $\hat{\ddot{y}}$ can be obtained as a sum of the contribution of all $n$ modal responses:

$$
\widehat{\ddot{y}}_{k}=\sum_{i=1}^{n} \widehat{\ddot{y}}_{k, i}=\sum_{i=1}^{n} V_{i} z_{m, k, i}
$$

where $V$ represents a matrix with the mode shapes of the $n$ vibration modes in the columns.

Considering that a reasonable number of accelerometers is installed, it is possible to increase the resolution of each mode shape (eigenvector) along the turbine support structure through a cubic spline interpolation. With this operation, the $V$ matrix from equation (3) can be replaced for a new $V_{\text {interp }}$ matrix (with the interpolated mode shapes) in order to estimate the modal acceleration at any point of the structure. It should be noted that a suitable number of sensors should be installed at the structure in order to accurately interpolate the tower mode shapes. Since the motion of the support structure is mainly driven by the $1^{\text {st }}$ and $2^{\text {nd }}$ vibration modes, the 3 considered levels of measurement guarantee a good measurement resolution for these modes.

At this point, the estimated modal acceleration responses have to be integrated into modal displacements $\hat{y}$. In that sense, the double integration of the acceleration 
time series in the frequency domain is performed. During this step, a suitable cut-off frequency should be used to avoid numerical errors due to the magnification of the amplitude of the very low frequency range of the spectrum. Once an estimation of the displacements of the turbine support structure is obtained, it is possible to compute equivalent forces imposing the same deformation according to:

$$
F(z)=K \hat{y}(k)
$$

where $z$ is referred to the height along the wind turbine tower and $K$ represents the stiffness matrix of the tower. Since the tower structure can be assumed as a simple cantilevered beam, the matrix $K$ can be constructed through simple methods, once the structural characteristics (variation of the bending stiffness along the height) are known.

After obtaining the equivalent forces along the tower height, the stress at any height of the support structure can be evaluated.

Although this procedure allows estimating the stress condition at any point of the wind turbine support structure, it should be noted that the accuracy of the results is dependent on the double integration process. In that sense, the frequency cut of the filter used during this step should be able to avoid numerical errors arising from the very low frequency range and, at the same time, cannot despise the frequency content due to the wind action and due to the structural resonances.

\subsection{Results}

The proposed procedure was validated through a numerical example of the wind turbine [8] using the HAWC2 aeroelastic code [9].

This procedure was then applied to the monitored 2.0 MW wind turbine. For this application, 3 levels of measurement were used (the sensors from the foundation were not considered). This procedure is illustrated with a real measured $10 \mathrm{~min}$ acceleration time series. In this example, the procedure was used together with the SSIDATA modal identification algorithm.

The 10 min setup is referred to an operating situation, under strong wind conditions (mean wind speed of 20.6 $\mathrm{m} / \mathrm{s}$ ). During this period, the turbine operated at its rated rotor speed (around $16.5 \mathrm{rpm}$ ) and the blades pitch angle varied between $9^{\circ}$ and $28^{\circ}$, in order to control the rotor torque. The acceleration time series collected by the dynamic monitoring system in the FA direction are presented in Figure 10.

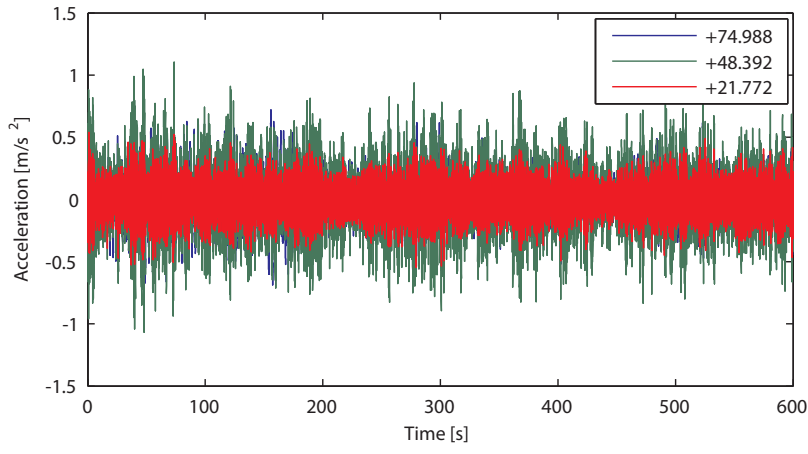

Figure 10. Acceleration time series in FA direction

The power spectral density of the acceleration time series is shown in Figure 11. In this figure, the frequency of the $3 \Omega$ and $6 \Omega$ harmonics is indicated by the vertical dashed lines. From this figure, it is observed that acceleration response is dominated by the $1^{\text {st }}$ and $2^{\text {nd }}$ tower bending modes ( $1 \mathrm{FA}$ and $2 \mathrm{FA}$ ), together with the $3 \Omega$ and $6 \Omega$ harmonics. It is also interesting to note the difference in the relative importance of the two modes in the acceleration response at the different measurement levels. While the acceleration at the top of the support structure is clearly dominated by the 1 FA mode, the acceleration at the other two levels is mainly driven by the 2 FA mode. It is visible that, at the top of the tower, the resonance peak from the 2 FA mode is practically unnoticed. This situation is justified by the almost inexistence modal amplitude at this level for the $2^{\text {nd }}$ tower bending modes (Figure 5).

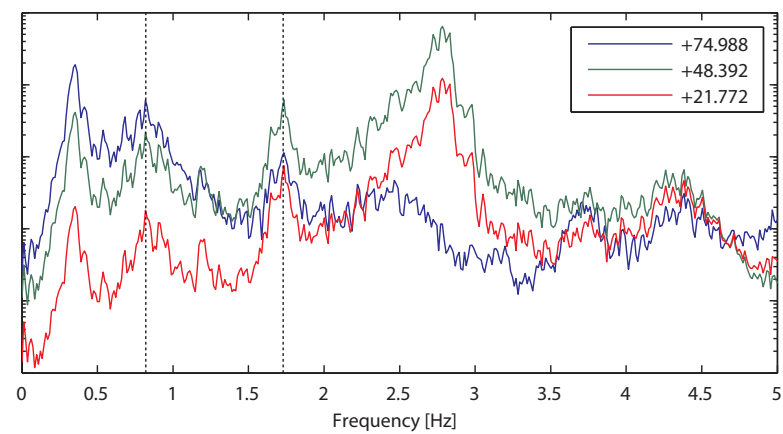

Figure 11. Spectra of the acceleration time series in FA direction (the dashed lines indicate the frequency of the $3 \Omega$ and $6 \Omega$ harmonics)

For the double integration process, a filter similar to the one introduced in Figure 12 was implemented. The use of this filter aims at reducing possible numerical errors from the very low frequency range which would be amplified during the integration. Nevertheless, the $f_{c 1}$ and $\mathrm{f}_{\mathrm{c} 2}$ constants of the filter have to consider that the main energy content of the wind action is located at the very low frequency range. In that sense, $f_{c 1}$ and $f_{c 2}$ were defined as, respectively, $0.02 \mathrm{~Hz}$ and $0.10 \mathrm{~Hz}$. These values were decided based on numerical tests performed with the HAWC2 code [9] and presented a good compromise between the amplification of possible low frequency errors and loss of wind energy from the low frequency region. Nevertheless, a suitable procedure to tune these parameters would be the temporary installation 
of sensors (e.g. strain gauges) to measure the quasi-static component of the displacement at an arbitrary section. Filter window

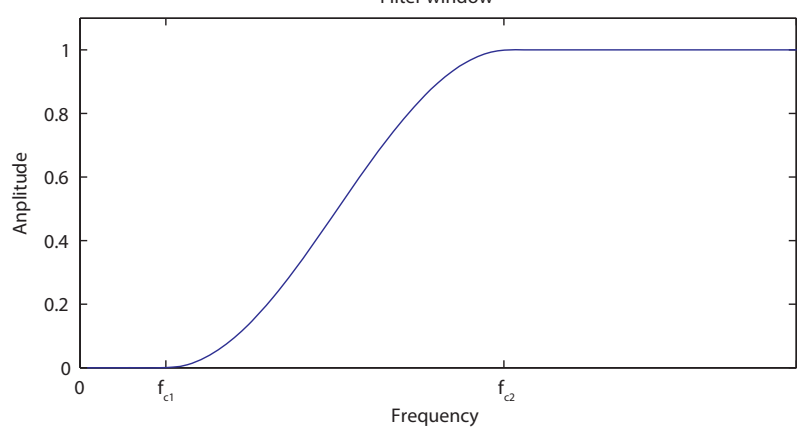

Figure 12. Filter window implemented for the double integration process of the acceleration time series

Before the integration process, the acceleration time series are multiplied by a time window with the aim of softening the start and end of the time series and thus reducing aliasing problems. These initial and last parts are then disregarded in the integrated displacement time series.

Figure 13 shows the obtained displacements at the measurement levels. As expected, the displacements are higher at the top, in opposition to what was obtained with the acceleration (Figure 10), since the displacements are mainly driven by the $1^{\text {st }}$ tower bending mode. In this figure, the transient initial and last parts are indicated by the vertical dashed lines and correspond to 30 seconds in each part.

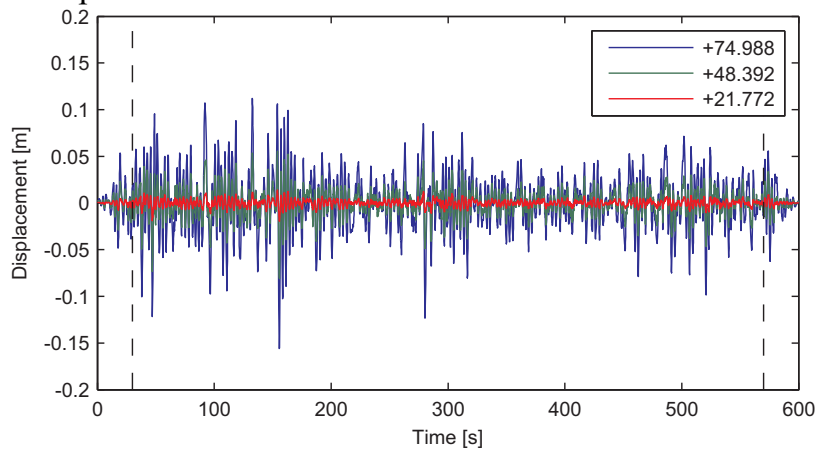

Figure 13. Displacement time series in FA direction (the vertical dashed lines indicate the disregarded initial and last part)

Once the displacements along the turbine support structure are obtained, it is possible to estimate the stress condition at any level of the tower. As an example, the bending stress at the foundation level was computed. The result is presented in Figure 14.

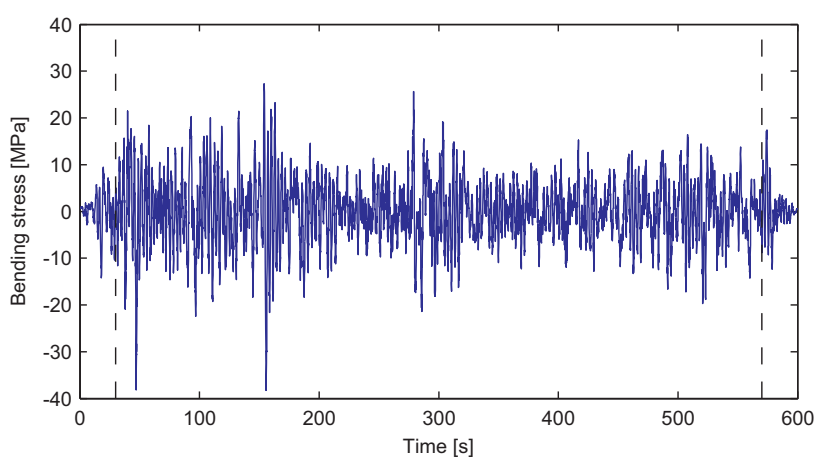

Figure 14. Bending stress at the foundation level in FA direction

At this point, the load cycles from the defined 540 seconds (600-2x30) bending stress time history are computed and the damage due to the setup can be evaluated. The Rainflow method was implemented in the monitoring system to count the load cycles, as it is the most used procedure in the standards. Figure 15 shows the obtained histogram of the load cycles. The computed accumulated damage during a representative period can then be extrapolate to estimate the remaining fatigue lifetime of the turbine support structure.

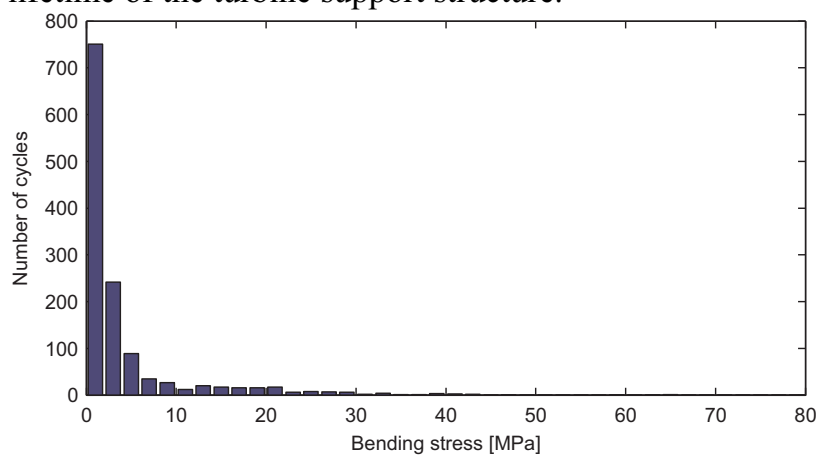

Figure 15. Histogram with the number of bending stress cycles

\section{Optimization of the dynamic monitoring system}

The methodology illustrated in the previous sections requires the installation of several accelerometers along the turbine support structure. In the case of the presented monitoring system, 3 levels were used. With the aim of reducing the installation cost of this kind of monitoring system in several wind turbines at a wind farm, an optimization of the monitoring system is proposed.

This optimization is based on the virtual sensors technique. Assuming that the motion of a structure is mainly driven by resonance of its vibration modes, it is possible to estimate the acceleration at any unmeasured position if the configuration of the $n$ vibration modes is known at that position. With this technique, it is possible to estimate the acceleration at locations where the installation of measurement devices is difficult or even impossible (such as under water locations of turbine offshore foundations).

In that sense, the $V$ matrix from equation (3) is replaced by a new $V_{v . s .}$ with the mode shapes containing both the measured and unmeasured positions: 


$$
\hat{\hat{y}}_{k}=\sum_{i=1}^{n} \hat{y}_{k, i}=\sum_{i=1}^{n} V_{v . s .} Z_{m, k, i}
$$

Wind turbines are usually equipped with accelerometers at the nacelle level. Thus, it would be interesting to use this single sensor to perform both the modal tracking and fatigue monitoring. However, the $2^{\text {nd }}$ tower bending mode has an almost inexistent modal amplitude at the tower top and, for that reason, its identification is practically impossible with only this sensor. Considering the importance of this mode to assess possible structural problems at the foundation, an additional second level of measurement is required. It was thus decided to use the top and the $+48.392 \mathrm{~m}$ level for the optimization layout of the monitoring system.

During a training period, a representative configuration of the main vibration modes was defined. In addition, representative configurations of the operational deflection shapes of the tower structure vibrating under the integer multiples of the $3 \Omega$ harmonic excitation were also identified. Considering that the procedure introduced in section 4.1 interprets the stationary response due to the harmonic excitation as a resonance motion, it is also possible to estimate the acceleration response due to the harmonics at unmeasured levels.

This methodology is illustrated with two examples: one related to a time setup corresponding to a nonoperating event, while the other is the one already presented in section 4.2.

The setup corresponding to a non-operating event, in which the turbine was parked, is related to a period of strong wind conditions. During this period, the wind speed varied considerably, between $5.6 \mathrm{~m} / \mathrm{s}$ and $32.1 \mathrm{~m} / \mathrm{s}$. The acceleration time series collected in the FA and SS direction are presented in Figure 16.

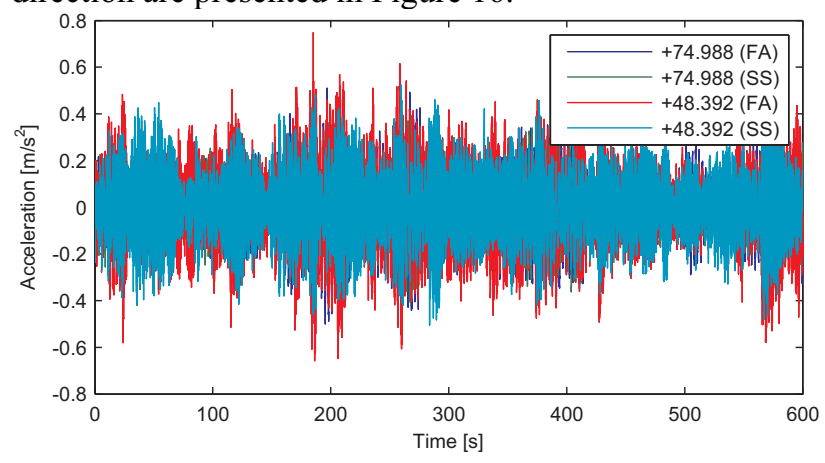

Figure 16. Acceleration time series during a non-operating event under strong wind conditions

This data (from sensors S5, S6, S8 and S9) was then processed in order to estimate the acceleration at the position of the S3 and S4 sensors, respectively, the FA and SS direction at the $+21.722 \mathrm{~m}$ level. The estimation in both directions is presented in Figure 17, alongside with measured accelerations.

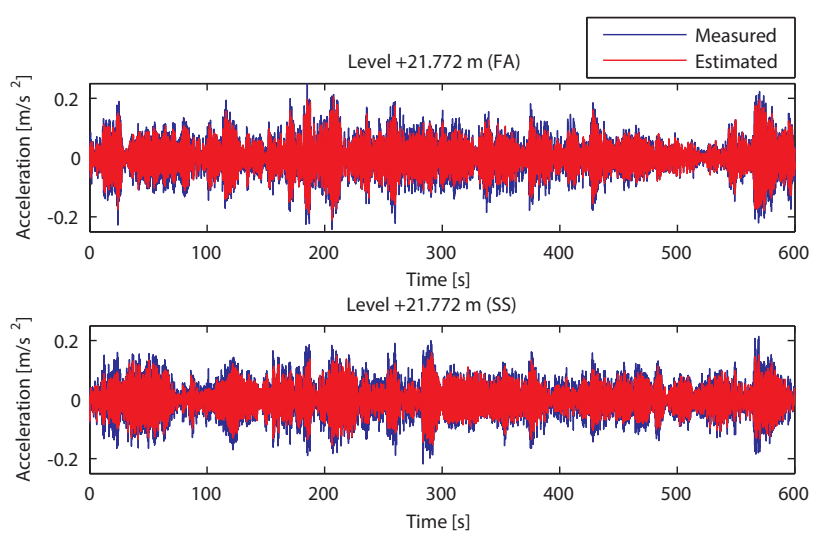

Figure 17. Measured and estimated acceleration time series at $+21.772 \mathrm{~m}$ level during a non-operating event

As can be seen, a good agreement was obtained between the measured acceleration signal and the estimated one. Figure 18 shows a zoom of the signals presented in Figure 17, where the good coherence can be assessed in a greater detail.
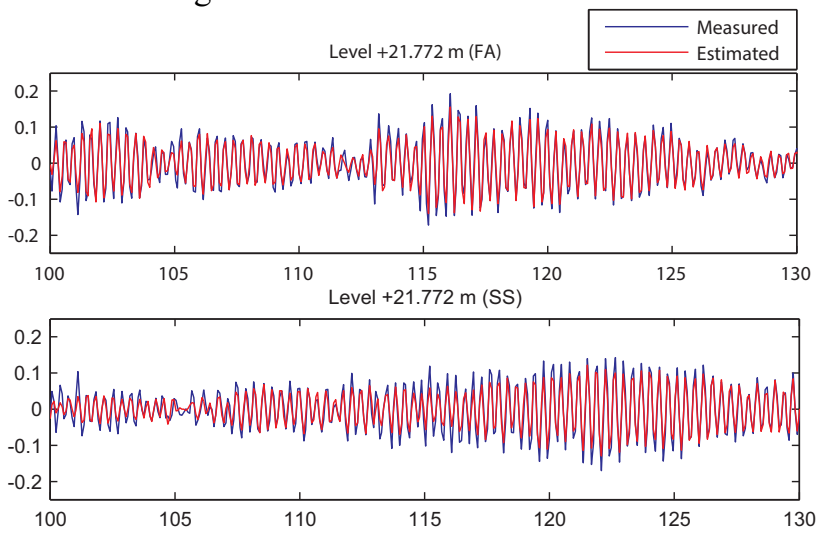

Figure 18. Zoom of the measured and estimated acceleration time series at $+21.772 \mathrm{~m}$ level during a non-operating event

The second setup is referred to the same $10 \mathrm{~min}$ event already introduced in section 4.2. Unlike the previously presented setup, the stationary response due to the harmonics excitation represents an important part of the structure motion and, for that reason, the operational deflection shapes of the tower structure vibrating at the harmonics frequency needs to be considered. Figure 19 shows the measured acceleration at $+21.772 \mathrm{~m}$ level and the estimated time series at this level considering only the top and +48.392 m measurement levels. 

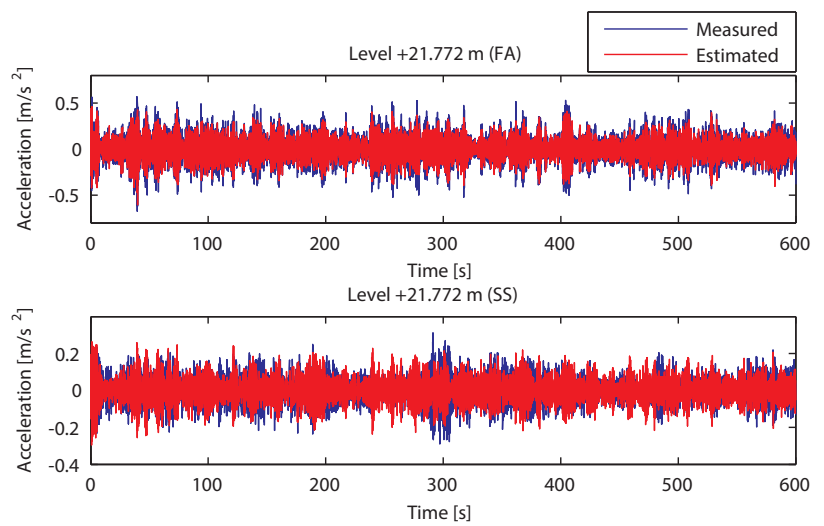

Figure 19. Measured and estimated acceleration time series at $+21.772 \mathrm{~m}$ level during an operating event

Once again, a very good agreement between the real and estimated acceleration time series is noticed. A zoom of $30 \mathrm{~s}$ of the time series is presented in Figure 20, where this good agreement is highlighted.
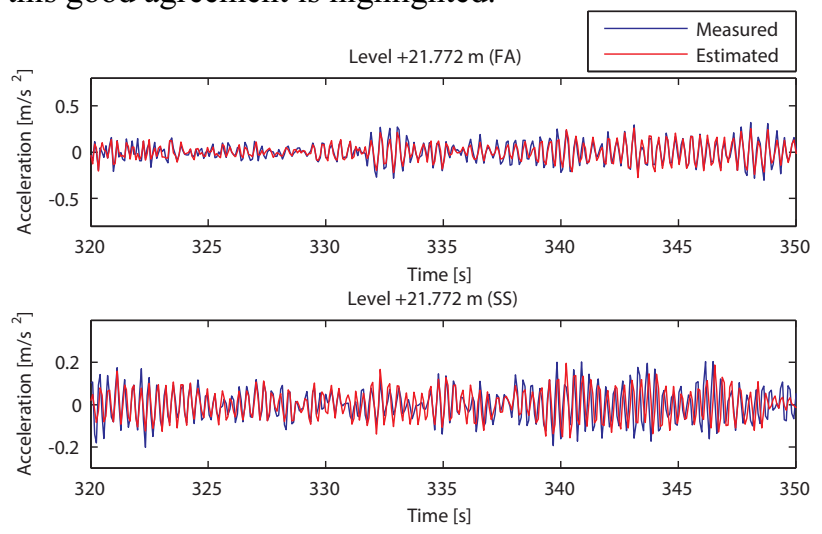

Figure 20. Zoom of the measured and estimated acceleration time series at $+21.772 \mathrm{~m}$ level during an operating event

\section{Conclusions}

The paper describes the dynamic monitoring system project of a 2.0 MW wind turbine. Throughout the paper, the two modules of the system (modal tracking and fatigue monitoring) were introduced and some results were presented, showing the suitability of the system to be implemented in onshore and offshore wind turbines. In addition, the estimation of the acceleration time series at unmeasured locations was also addressed, enabling the optimization of the measurement layout.

In future developments, the investigation of the possibilities achieved with the system optimization will be addressed for both modal tracking and fatigue assessment.

\section{Acknowledgments}

The authors would like to acknowledge: (1) the Ph.D. Scholarship (SFRH/BD/79328/2011) provided by FCT to the first author; (2) the support provided by FCT to ViBest/FEUP in the area of Continuous Dynamic Monitoring and (3) the support given by INEGI, the wind turbine manufacturer Senvion and the wind turbine owner Cavalum.

\section{References}

1. F. Magalhães, Á. Cunha, Mechanical Systems and Signal Processing, 25, 1431-1450 (2011)

2. G. Oliveira, W. Weijtjens, G. D. Sitter, F. Magalhães, Á. Cunha, E. Caetano, C. Devriendt, RENEW-1 ${ }^{\text {st }}$ International Conference on Renewable Energies Offshore (2014)

3. G. Oliveira, F. Magalhães, Á. Cunha, E. Caetano, IOMAC'15 (2015)

4. B. Peeters, G. Roeck, Mechanical Systems and Signal Processing, 13(6), 855-878 (1999)

5. P. Overschee, B. Moor, Subspace Identification for Linear Systems. Theory, Implementation, Applications (Kluwer Academic Publishers, 1996)

6. B. Peeters, H. Auweraer, IOMAC'05 (2005)

7. F. Magalhães, Á. Cunha, E. Caetano, Mechanical Systems and Signal Processing, 25(2), 316-32 (2009)

8. G. Oliveira, F. Magalhães, A. Cunha, E. Caetano, COMPDYN 2015 (2015)

9. T. Larsen, A. Hansen, How 2 HAWC2, the user's 